\title{
Globe
}

Revue internationale d'études québécoises

\section{Le grand récit des arts de la scène au Québec}

\section{Erin Hurley}

Volume 11, numéro 2, 2008

Les arts de la scène au Québec

URI : https://id.erudit.org/iderudit/1000519ar

DOI : https://doi.org/10.7202/1000519ar

Aller au sommaire du numéro

Éditeur(s)

Globe, Revue internationale d'études québécoises

ISSN

1481-5869 (imprimé)

1923-8231 (numérique)

Découvrir la revue

Citer ce document

Hurley, E. (2008). Le grand récit des arts de la scène au Québec. Globe, 11(2),

11-22. https://doi.org/10.7202/1000519ar d'utilisation que vous pouvez consulter en ligne.

https://apropos.erudit.org/fr/usagers/politique-dutilisation/ 


\title{
INTRODUCTION. \\ LE GRAND RECIT DES ARTS \\ DE LA SCENE AU QUÉBEC
}

\begin{abstract}
ERIN HURLEY
Université McGill

Les arts de la scène constituent l'un des secteurs les plus dynamiques de la culture québécoise contemporaine. Depuis une vingtaine d'années, l'envergure et la qualité des festivals consacrés aux pratiques scéniques ainsi que le remarquable succès de plusieurs compagnies à l'étranger ont témoigné éloquemment de l'insertion des artistes québécois dans les circuits internationaux. Pour ne mettre en relief que quelques exemples, mentionnons qu'en 1990 le prestigieux Brooklyn Academy of Music consacrait une portion de son festival annuel Next Wave aux "New Currents from Montreal". On retrouvait, parmi les invités, le Théâtre Repère (dirigé à l'époque par Robert Lepage), Carbone 14, la réputée soliste montréalaise Margie Gillis et la compagnie de danse contemporaine de Ginette Laurin, O Vertigo. En 1998, Philadelphie recevait O Vertigo et Margie Gillis, accompagnées cette fois par le Théâtre des Deux Mondes, et mettait à l'affiche une pièce de Michel Lemieux et Victor Pilon, de la compagnie 4d arts. Depuis des années, les spectacles du Théâtre Sans Fil et du Théâtre de l'Eil sont accueillis chaleureusement par des festivals internationaux consacrés aux marionnettes et au théâtre jeune public ${ }^{1}$. Mais la compagnie québécoise dont le rayonnement est le plus grand reste sans doute le Cirque du Soleil. Avec ses quatre spectacles fixes à Las Vegas (sans compter ceux de la Floride, de New York et de Macao), ses six tournées mondiales simultanées ainsi que ses deux

$$
++
$$

1. Claude DES LANDES, «D'une entreprise nationale au rayonnement planétaire *, LAnnuatre theatral, $n^{\circ} 27$, printemps 2000, p. 31-43; Hélène BEAUCHAMP, "Jeunes d'ici, jeunes d'alleurs: questions de culture(s) et de theátre*, L'Annuatre theâtrath $n^{\circ} 27$, printemps 2000 , p. 55-67. 
spectacles en arénas, force est de constater que le territoire du "cirque québécois" qu'il représente dépasse de beaucoup le Québec².

Comme la diffusion à l'étranger est liée (du moins en partie) à l'offre de réciprocité et que le Québec se veut un lieu-phare et un tremplin pour les artistes de la scène, maints festivals culturels se déroulent ou se déroulaient dans la métropole chaque année: le Festival de théâtre des Amériques (rebaptisé en 2007 le Festival TransAmériques), le défunt Festival international de nouvelle danse, le Festival Voix d'Amériques (festival de spoken word ${ }^{3}$ ), le Festival St-Ambroise Fringe de Montréal (festival de thêâtre amateur), le Festival Juste pour rire, les FrancoFolies (festival de musique francophone) et le Festival International de jazz de Montréal (et j'en passe).

Ce dossier consacré aux arts de la scène au Québec reflète cette réalité d'une production culturelle de plus en plus mondialisée et structurée par réseautage. Plusieurs des contributions rassemblées ici portent sur des créateurs, des troupes et des spectacles qui ont circulé et se sont fait une place permanente sur la scène internationale. Michel Tremblay, Denis Marleau, Robert Lepage, Carbone 14 et le Cirque du Soleil comptent parmi les représentants du theâtre québécois (pour ne pas dire parmi les "produits" culturels québécois) les plus visibles et recherchés à l'étranger, au point où les gouvernements successifs du Québec les reconnaissent comme des "ambassadeurs culturels" du Québec. Si ces artistes bénéficient d'un vaste rayonnement et d'une reconnaissance internationale, ils ont également suivi un parcours similaire depuis des zones marginales vers un centre culturel. Comme le soulignent nos collaborateurs, c'est dans la rue que naissent Carbone 14 et le Cirque du Soleil. D'ailleurs, avant de s'installer dans leurs locaux respectifs de Montréal et de Québec, Carbone 14, le Cirque du Soleil et la compagnie Ex Machina de Robert Lepage étaient plutôt itinérants. Pour leur part, les pièces qui composent le Cycle des belles-sceurs de Michel Tremblay, vite devenues, depuis les années 1970, des classiques de la dramaturgie québécoise, se nourrissent de la marginalité et de l'excentricité de la Main. Qui plus est, les artistes dont traitent nos collaborateurs ont fortement contribué à créer la marque du théâtre québécois contemporain désormais reconnue au Canada anglais et à l'étranger. Bien sûr, le milieu

$$
++
$$

2. Je voudrass signaler la parution prochaine du dossıer "Le Quebec à Las Vegas» de L'Annuaire theàrrah $n^{\circ} 43$, prıntempslété 2009, et rout particulièrement Particle de Lours Patrick LEROUX, «Zumaniry: la spectacularisatıon de l'intıme, ou le pari impossible d'authenticicé au Cirque".

3. Pour en savoir plus sur le spoken word, on consultera l'ouvrage de Vicroria STANTON er Vincent TANGuELY, Impure. Reinventing the Word. The Theory, Practice, and Oral History of 'Spoken Word' in Montreal, Montreal, Conundrum Press, 2001 . 
actuel des arts de la scène - dans la pratique comme dans la recherche universitaire - est vaste et dynamique, et je m'en tiendrai donc, pour le bénéfice des lecteurs moins familiers de ce champ d'étude, à un bref tour d'horizon des moments et des figures clefs ainsi que des principales sources qui permettent d'éclairer les contributions ici rassemblées.

\section{UN GRAND RÉCIT}

Baromètre sensible de l'actualité, le monologue comique, dont l'historien Robert Aird retrace l'histoire du XIX"e siècle jusqu'à la fin du XXe siècle, "met l'accent sur les travers de ses contemporains, de son époque, souvent en racontant des épisodes de la vie quotidienne". Retraçant les liens étroits qu'entretiennent les personnages comiques et le peuple québécois à travers différents lieux de diffusion du monologue comique, Aird souligne l'atténuation frappante du récit d'antan en faveur du régime commercial contemporain de one-liner. Du coup, ce sont aussi la critique sociale et le lien entre scène et société qui tendent à disparaître. Il y a deux raisons significatives d'ouvrir ce dossier par l'article de Robert Aird: non seulement s'agitil de la seule contribution qui porte sur la période antérieure à la Révolution tranquille, mais c'est aussi l'article qui résume le plus clairement le grand récit identitaire des arts de la scène québécois, dont les origines marginales et folkloriques, étroitement liées à la sociéré, finissent par être oubliées au profit d'une approche américaine mainstream dépourvue de critique sociale.

Il n'est pas étonnant que ce dossier mette l'accent sur la production culturelle contemporaine, celle où l'on reconnaît et promeut la spécificité des arts de la scène au Québec. Un tel accent sur la période qui s'ouvre avec les années 1960 est conforme au grand récit identitaire des arts de la scène québécois, du moins tel qu'il circule et est repris dans la plupart des ouvrages sur le sujet. Parler du théâtre "québécois", en fait, c'est parler du théâtre depuis la Révolution tranquille, dans la mesure où l'histoire du théâtre québécois semble parfois calquée sur celle de l'éclosion et de l'émancipation de sa société, l'une et l'autre étant en «développement". Faisant du

$$
+++
$$

\footnotetext{
4. Ce n'est pas que les publications sur le thêatre de la pré-Révolution tranquille soıent inexistantes, mais elles demeurent minoritaires dans le domaine. Les ouvrages de Jean-Marc Larrue, d'André-G. Bourassa et de Gilbert David, par exemple, témoignent de l'intérêt et de la vitalité de l'histoire du théâtre québécoss Jean-Marc LaRRUE, Le théatre a Montréal à la fin du XXX' siècle, Montréal, Fides, 1981 ; André-G. BOURASSA et Jean-Marc LARRUE, Les nuts de la "Matn" : cent ans de spectacles sur le boulevard Saint-Laurent (1891-1991), Montréal, VLB, 1993; André-G. BOURASSA, "Les visiteurs au pouvoir: le théâtre au Québec, 1850-1879. $1^{\text {"x }}$ partie», Bulletin d'hstoire polinque, vol. 13, $n^{\circ} 2$, hiver 2005, p. 191-216; André-G. BOURASSA, «Les visiteurs au pouvoir: le thêtre au Québec, 1850-1879. $2^{\complement}$ partie ", Bulletin d'historre politique, vol. 13, $n^{\circ}$, printemps 2005, p. 123-145; Gilbert DAVID, “Un thêatre à vif: écritures dramatiques ec pratiques scéniques au Québec, de 1930 à 1990n, thèse de doctorax, Département d'études françaises, Université de Montréal, $1995,451 \mathrm{f}$.
} 
thêâtre l'homologue de la société, cette histoire reprend les conventions narratives de ce que Jocelyn Létourneau appelle «le grand récit de l'identité québécoise», un récit familier aux lecteurs de Globe et dans lequel le Québec émerge de la Grande noirceur coloniale au moment de la fameuse Révolution tranquille pour atteindre maturité politique, gouvernance et connaissance de soi en tant que Sujet-Nations. Ainsi, par exemple, Madeleine Greffard et Jean-Guy Sabourin ouvrent leur introduction au thêâtre québécois avec le constat que l'activité théâtrale sur le territoire a connu un lent développement qui correspond à celui de la société canadienne-française: «ll a pourtant fallu plus de trois siècles pour que le théâtre joué sur le territoire du Québec devienne le théâtre de la société québécoise ${ }^{6}$." Ils nous rappellent que les pièces montées au XIXe siècle et jusqu'aux années 1950 appartenaient en majorité au répertoire français ou étaient des divertissements populaires en provenance des États-Unis. D'après Jean Cléo Godin et Dominique Lafon, qui reprennent une histoire semblable du thêâtre québécois dans l'introduction de leur ouvrage, Marcel Dubé (1930-...) «avait identifié ce milieu [d'une société trop longtemps dominée et humiliée] aux quartiers populaires de Montréal et à une sorte de poésie valorisante du quotidien ${ }^{7}$ ». Les pièces réalistes de Dubé auraient ainsi ouvert la voie à une nouvelle génération de dramaturges plus audacieux dans leur rapport à la langue et dans le choix de leurs thèmes, parmi lesquels figurent Marie Laberge (1950-...), Michel Tremblay (1942-...), Jean-Claude Germain (1939-...) et Françoise Loranger (1913-1995). C'est pourtant le "miroir effrayant ${ }^{8}$ " offert par Les belles-seeurs (1968) de Michel Tremblay qui signale la fin du théâtre colonial. Pour maints arristes, critiques et universitaires, ces belles-sœurs donnent naissance à une nouvelle esthétique, à un nouveau langage théâtral et à un nouveau type de thêâtre: le nouveau théâtre québécois, qui, selon Michel Bélair, se veut marginal par rapport à la tradition du thêâtre poétique, importé, "high art ${ }^{9}$ ". Dans le tout premier ouvrage universitaire sur le théâtre québécois proprement dit, Théâtre québécois I, Jean Cléo Godin et Laurent Mailhot repren-

$++4$

5. Jocelyn LetOURNeAU, "The Current Great Narrative of Québecois Identity", SAQ (Soush Atlantic Quarterly), vol. 94, $n^{\circ} 4$, automne 1995 , p. 1042.

6. Madeleine GREFFARD et Jean-Guy SABOURIN, Le theâtre québecois, Montréal, Boréal, 1997.

7. Jean Cléo GODIN er Domınique LAFON, Dramaturgies québecouses des années quatre-vingts, Ottawa, Leméac, 1999, p. 7 .

8. "Miroir effrayant" vient de la critique louangeuse de la pièce par Jean-Claude GermaIN, "Les belles-sarurs: une condamnation sans appel ", Le Petst Journal, 8 septembre 1968, p. 10.

9. C'est ainsi que Michel Bélair, critıque au Devorr à l'époque, résume les modıficarıons apportées à la dramaturgie par Tremblay er ses compatriotes (Michel BÉLAIR, Le nouveau theâtre quebecoss, Montréal, Lemeac, 1973). 
nent grosso modo le propos de Bélair. Ils suggèrent que ce qui fait le théâtre "québécois", c'est qu'il "reflète et identifie notre milieu".

Des trois genres littéraires, c'est peut-être le thêâtre qui, par ses caractéristiques particulières, est le plus lié au milieu. Non seulement parce qu'il le reflète, s'en nourrit, l'attaque, mais parce que son succès, son existence, dépendent directement de la réception que lui donnera ce milieu. Le théâtre est placé entre deux miroirs aux écrans très rapprochés ${ }^{10}$.

Ce "nouveau théâtre québécois" fonde le drame dans le récit d'histoires vécues, dans une langue et des personnages «indigènes", afin de provoquer une prise de conscience nationale chez les spectateurs. Les belles-sceurs, par exemple, baigne dans un univers urbain, féminin et joualisant. Jacques Cotnam perçoit lui aussi dans ce théâtre une réalisation du but d'une expression dramaturgique qui capte la québécité émergente de l'époque: "Michel Tremblay est en train de créer une dramaturgie nationale authentique, qui reflète les frustrations accumulées depuis trois cents ans et la révolte qu'elles ont finalement provoquée ${ }^{11}$."

En examinant ainsi l'historiographie canonique de la naissance du théâtre québécois, je ne cherche pas à laisser entendre que ce récit du "progrès" manque de justesse. Par contre, une reprise aussi fréquente témoigne non seulement du fait qu'il soit accepté et largement répandu, mais aussi de sa force de persuasion. Les archives de diffusion et de publication documentent et confirment de façon indiscutable la prépondérance de pièces étrangères sur la scène au Québec avant les années 1950. La Révolution tranquille et sa réitération de l'identité s'accompagnent d'une vague d'ouvrages qui récupèrent les pièces "indigènes", surtout du XIX siècle, ou qui documentent la vigueur du théâtre québécois depuis la deuxième moitié du $\mathrm{XX}^{e}$ siècle $^{12}$. Des statistiques complémentaires enregistrent l'augmentation en

$$
+\div
$$

10. Jean Cléo GoDIN et Laurent MAILHOT, Théatre quebecoss 1, Introduction à dix dramaturges contemporains, nouvelle édition, Montréal, Hurtubise HMH, 1988 [1970], p. 20.

11. Jacques COTNAM, Le théâtre québecois, instrument de contestanon sociale et politique. Etudes litteratres, Montréal, Fides, 1976, p. 67. Même si les pièces qui composent le Cyck des belles-sczurs de Tremblay demeurent pour la critique le symbole de la marginalité revendicarrice des années 1970, il faut aussı tenir compte de plusieurs créations collecrives féministes importantes au Québec qui ont partagé le mème esprıt revendicateur. Voir LE THÉ̉TRE DES CUISINES, Môman travaille pass, a trop d'ouvrage, Montréal, Éditions du remue-ménage, 1976; Marie-Louise DiON er Louise PORTAL, Où en est le miroir?. Montréal, Éditions du semue-ménage, 1979; LE THEÁTRE DU HORLA, La Vraie Vie des masqués, Montréal, Éditions du remue-ménage, 1979; Dominique GaGnon, louise LAPrade, Louse LeCavalier, Pol Pelletier, $A$ ma mère, à ma mère, d̀ ma mère, à ma voissne, Montréal, Éditions du remue-ménage, 1979.

12. Jean HaMelin, Le renouveau du theáste au Canada franças, Montréal, Les Éditions du Jour, 1961 ; Baudoin BURGer, Lactivité shéărale au Quebec (1765-1825), Montréal, Parti pris, 1974; Étienne-F. Duval [dir.], Anthologie thematique du théatre québecous au XIX' stècle, Montréal, Leméac, 1978. 
flèche de pièces québécoises montées à Montréal après la Révolution tranquille ${ }^{13}$. Cette approche historique du théâtre québécois a l'avantage d'une admirable clarté et met en relief les conditions de la production théâtrale (ainsi que celles de la musique et de la danse) de part et d'autre de cette division temporelle. Le contraste, frappant, est efficace sur le plan pédagogique (surtout pour les étrangers et les non-spécialistes en études québécoises) et irrésistible sur le plan narratif, et, en toute justesse, célèbre le succès du thêâtre québécois. Or, il faut souligner qu'un tel récit, consciemment réducteur, dépend tout de même d'une connaissance beaucoup plus nuancée des événements et des mouvements chez les spécialistes du domaine ainsi que dans les pages des revues québécoises consacrées aux arts de la scène, où publient le plus souvent ces spécialistes.

Quoi qu'il en soit, le succès est indéniable. En se dotant d'institutions thêatrales, en se professionnalisant et se nationalisant, le théâtre québécois connaît un essor fulgurant sur une très courte période de quarante ans. Le milieu théâtral s'institutionnalise: le rideau se lève sur la première production du Théâtre du Nouveau Monde en 1951; le Conseil des arts de la région métropolitaine commence à octroyer des subventions en 1956; le Centre d'essai des auteurs dramatiques est fondé en 1965 (il enregistre sa nouvelle raison sociale, le Centre des auteurs dramatiques, en 1991); la Place des Arts s'érige en 1963 au centre-ville de Montréal tandis que Québec inaugure le Grand Thêatre de Québec en $1971^{14}$. Le théâtre se professionnalise: le Conservatoire de musique et d'art dramatique, un réseau d'écoles des arts de la scène, dont deux de théâtre et sept de musique, est fondé en 1942; depuis 1961, l'École nationale de théâtre du Canada à Montréal offre une formation professionnelle en jeu, en écriture dramatique, en scénographie et en production; les arts de la piste s'enseignent à l'École nationale du cirque à Montréal (fondée en 1981). Le milieu s'institutionnalise, avec la fondation de sociétés des arts de la scène comme l'Union des artistes (1937), et de sociétés et d'organes de la critique universitaire. La Société de l'histoire du thêâtre québécois, devenue en 1992 la Société québécoises d'études thêâtrales, s'organise en 1976 et publie une revue scientifique, L'Annuaire

$++4$

13. Pierre LAVOIE, "Québec/bilan tranquille d'une révolution thêâtralen, Cahters de thêâtre Jeu, n 6, 1977, p. 47-61; Josette FERAL, "Pratiques culturelles au Québec: le theâtre et son public", Etudes littératres, vol. 18, $\mathrm{n}^{0} 3,1985$, p. 191-210; Elaine F. NARDOCCHIO, "1958-1968: Ten Formative Years in Québec's Theatre History", Canadzan Drama/L'Art dramatique canadien, vol. 12, $\mathrm{n}^{\circ} 1,1986, \mathrm{p} .33-63$.

14. Voir Helène BEAUCHAMP, "Les thêâtres. Lieux de représentation et lieux de création thêâtrale au $\mathrm{XX}^{*}$ siècle *, Hélène BEAUCHAMP ec Gilbert DAVID [dir.], Theátre québecoss et canadiens-françats au XX' stècle, Sainte-Foy, Presses de l'Unıversıté du Québec, 2003, p. 33-58. 
théâtral; les Cahiers de théatre Jeu deviennent en 1976 le principal observateur du théâtre québécois peu après leur premier numéro consacré à une troupe de jeune théâtre pratiquant la création collective: le Parminou. Le thêâtre se québécise: le Thêâtre d'aujourd'hui se donne pour mandat de produire des pièces québécoises; de grands écrivains et des maisons d'édition travaillent ensemble afin de traduire des pièces de répertoire en français du Québec, un effort auquel participent notamment Michel Garneau et Michel Tremblay's

L'œuvre de Michel Tremblay est située au centre de ce discours de "développement" du théâtre québécois, surtout ses pièces Les belles-seours (1968) et Hosanna (1970). Dans sa lecture attentive de trois productions de Hosanna (celle de 1975 au Warehouse Theatre de Winnipeg, celle de 1991 au Théâtre de Quat'sous et la plus récente production au Théâtre du Nouveau Monde, en 2006), Robert Schwartzwald examine la valeur symbolique et actuelle de cette marginalité vantée. Il s'interroge sur la manière dont Hosanna et son personnage de travesti nous parlent aujourd'hui différemment d'autrefois, et retrace les significations variables de la pièce dans un contexte social changeant, de plus en plus "normalisant" pour le couple homosexuel, alors que le discours politique revendique de moins en moins la marginalité du Québec. Rappelons par ailleurs que la publication du Nouveau théatre québécois (1973) de Michel Bélair fait date «doublement, d'abord en fixant l'expression qui allait désigner la production thêâtrale d'une génération et ensuite en qualifiant celle-ci de marginale [je souligne] ${ }^{16} "$. L'analyse de Robert Schwartzwald, qui relève les éléments significatifs de chacune de ces trois représentations, apporte un éclairage intéressant sur les enjeux de la représentation du sujet homosexuel et du Sujet-Nation au Québec, qui met en quelque sorte à l'épreuve le propos de Bélair énoncé il y a plus de trente ans: "La marginalité du nouveau théâtre québécois est irrémédiablement liée à la marginalité du contexte politico-social et culturel du Québec ${ }^{17}$."

\section{LESTHÉTIQUE DOMINANTE DES ANNÉES 1980}

Si l'on fait exception du théâtre de Tremblay, ce sont des metteurs en scène et des compagnies des années 1980 dont parlent les collaborateurs de ce dossier. À la suite de l'éveil politique et culturel des années 1960 et 1970, une

$+\div+$

15. Annie BRISSET, Sociocritrque de la traduction. Theâtre et altetrité au Québec (1968-1988), Longueuil, Le Préambule, 1990; Louise LADOUCEUR, Making the Scene. La traductson du theatre d'une langue officielle à l'autre au Canada, Montréal, Nota bene, 2005.

16. Yves Jubinvile, «Appel d'air. Regards obliques sur l'ınstitution théatrale au Québeç, Hélène BEAUCHAMP et Gilbert DAVID [dir.], op. ctt., p. 326-327.

17. Michel BELAIR, op. cit., p. 13. 
époque marquée par l'affermissement de l'institution littéraire québécoise ainsi que par l'essor de genres perçus comme étant spécifiquement québécois (la chanson québécoise, le nouveau théatre québécois), le théâtre des années 1980 semble prendre du recul par rapport au modèle dominant d'un theâtre de la québécité et insiste plutôt sur l'image visuelle ${ }^{18}$. Comme le prouvent les exemples du monologue comique et du théâtre de Tremblay cités plus haut, les arts de la scène au Québec se sont longtemps enracinés dans l'importance de la parole et dans une fonction d'identification sociale. Cependant, après l'échec du référendum de 1980, les arts de la scène abandonnent en quelque sorte le regard sociocritique et le mode réaliste afin d'embrasser de nouvelles formes d'expression et de renouveler le langage scénique. La critique sociale des années 1970 cède la place à un traitement plus prononcé de la forme, autant dans le milieu thêâtral qu'en danse. Les pièces d'une nouvelle génération de dramaturges, à laquelle appartiennent Normand Chaurette (1954-...), René-Daniel Dubois (1955-...) et Michel Marc Bouchard (1958-...), sont exemplaires d'un théâtre dont, écrivent Godin et Lafon,

la critique a été unanime à [...] reconnaître deux traits caractéristiques: l'abandon quasi total de la langue parlée populaire au profit d'une écriture plus standard (sans être "parisienne") et la fin d'un théâtre spéculaire et identitaire, auquel succèdent des récits à la fois plus éclatés et plus introspectifs's

Les pièces typées de cette décennie se distinguent par une autoréférentialité marquée, l'absence de projet collectif et la tendance baroque de l'écriture ${ }^{20}$. La thématique de la création y est omniprésente. Même Michel Tremblay le dramaturge le plus étroitement lié au théâtre identitaire des années 1970 change de cap: il souligne en quelque sorte ce recul avec L'Impromptu d'Outremont, qui quitte la cuisine des belles-soeurs et le "one-room expensive dump» de Hosanna pour le salon bourgeois où se réunissent les quatre sœurs Beaugrand.

C'est à cette même époque de relarive quiétude politique que se bâtit la réputation internationale de Carbone 14, une troupe de théâtre fondée en 1980 par Gilles Maheu (1948-...), son directeur artistique, scéno-

$$
++
$$

18. Louise VIGEANT, "Du réalisme à l'expressionisme. La dramaturgie québeccoise récente à grands tratts", Cahters de theatre Jeu, n' 58,1991 , p. 7-16.

19. Jean Cléo Godin et Dominique Lafon, op. cit., p. 12.

20. Voir le dossier «Dramarurgie québécoise (1980-1990) * de L'Annuaire theatrah n $\mathrm{n}^{\circ} 10$, automne 1991; Gılbert DAvid [dir ], Veilleurs de nutt 3. Bilan de la satson theatrale 1990-1991, Montréal, Les Herbes rouges, nos 195-196, 1991. 
graphe et chorégraphe principal. Ses créations privilégient le «texte performance" ("performance text») plutôt que le texte écrit, et font partie d'un mouvement théâtral qui déplace l'accent du réalisme populaire vers la performance hybride d'avant-garde. Durant les années 1980 , ce théâtre de l'image signé Carbone 14 et Robert Lepage prend son essor sur les marchés nationaux et internationaux des arts de la scène; ils misent sur la technologie et les images visuelles marquantes. Dans la réflexion sur l'esthétique dominante des années 1980, on ira jusqu'à parler d'une "ère de scénographes". Pour les auteurs de La face cachée du théatre de l'image, Chantal Hébert et Irène PerelliContos, l'appellation "le thêtre de l'image" indique "la primauté accordée à l'image scénique qui se trouve hissée, par une génération d'artistes très actifs à partir des années quatre-vingts, au rang où la tradition occidentale avait placé le texte dramatique ${ }^{21}$ \%. Maheu et Lepage développent des processus de création analogues quoique distincts, où le spectacle se compose dans l'espace scénique et non sur la page. Hébert et Perelli-Contos décrivent ainsi ses principes:

Cetre écriture scénique consiste en un assemblage, un brassage ou un bricolage d'objets, de paroles, de musiques, de sons, d'éclairages, de textes, de gestes, de mouvements, d'appareils technologiques, d'écrans, etc., bref, d'éléments disparates et hétérogènes qui s'offrent comme des ressources sensibles potentiellement exploitables tout au long de la création theâtrale. La combinaison, la recombinaison, le déplacement, le jeu de ces éléments permet la constitution de la matière ou texte spectaculaire, laquelle tient précisément dans le rapport intime que ces matériaux ou éléments scéniques établissent entre eux et avec l'espace thếtral ${ }^{22}$.

Dans sa contribution "De la rue à l'Usine $\mathrm{C}$ ", Adeline Gendron nous offre l'un des premiers articles retraçant le parcours d'une compagnie dont personne ne doute de l'importance historique. Situé au milieu du dossier, l'article de Gendron revient sur la tension entre marginalité et normativité qu'interroge Schwartzwald, et ce, par rapport aux lieux où se produit Carbone 14. Gendron souligne la marginalité et l'excentricité des débuts de Carbone 14 sur le plan générique, mais aussi sur celui de la formation, du public et des lieux de diffusion. Bien que Carbone 14 cherchât alors à investir

$$
+4
$$

21. Chantal HéberT et Irène PERELL-CONTOS, La face cachée du theâtre de limage, Quebec, Les Presses de l'Université Laval, 2001, p. 11.

22. Jbid. p. 9. 
les lieux publics avec des spectacles tenant à la fois du mime et du théâtre et offrant une critique de la société de consommation, les productions les plus récentes de la troupe profitent d'une extravagante scénographie et d'un rapport frontal avec l'assistance rendus possibles par son installation à l'Usine $\mathrm{C}$, centre de création et de diffusion multidisciplinaire.

À l'avant-garde de ce virage vers le formalisme et le corps se situe le travail du metteur en scène et fondateur du Théâtre Ubu, Denis Marleau (1954-...). Depuis sa création Merz opéra en 1989, la plupart des spectacles de la compagnie Ubu, fondée en 1982, sont présentés en Europe autant qu'au Québec et au Canada. Ancien directeur artistique du Théâtre Français du Centre national des arts du Canada à Ottawa (2000-2007), Denis Marleau a suivi les traces de Robert Lepage et de son prédécesseur, le metteur en scène André Brassard, jusqu'à tout récemment collaborateur attitré de Michel Tremblay. Son cas s'avère particulièrement révélateur des esthétiques en mouvance au cours des deux dernières décennies du XXe siècle: en effet, Marleau s'est fait connaître à travers ses représentations hybrides entre théâtre et nouvelle danse dans les années 1980, pour ensuite se concentrer de plus en plus sur l'immobilité et le texte dans les années 1990 et 2000. André-G. Bourassa note "le recours à la danse» de Marleau, qui a su intégrer les chorégraphies des maîtres de la nouvelle danse québécoise postmoderne, entre autres Édouard Locke, Daniel Léveillé et Ginette Laurin, dans ses mises en scène de pièces de répertoire européen au début de sa carrière ${ }^{23}$. L'article d'Ėve Irène Therrien, "De l'inanimé à l'anima. Le fil conducteur entre l'acteur et le spectateur chez Denis Marleau", nous introduit au style actuel d'un des grands metteurs en scène du théâtre contemporain, sujet privilégié de la critique universitaire pour ses spectacles parfois perturbateurs et son style bien distinct ${ }^{24}$, style qui semble d'ailleurs aux antipodes de celui des débuts. Les premiers efforts de Marleau visaient en effet à capter les pulsions intimes des personnages à travers l'expérience du corps, voire d'un corps aux mouvements inusités. Mais depuis les dix dernières années, l'accent est mis plutôt sur la voix, au détriment du corps. L'article de Therrien, justement,

$$
+4
$$

23. André-G. BouRASSA, "Scène québécoise. Permutations de formes et de fragments en danse, mime et thêatre", Études Ittteraires, vol. 18, $n^{\circ} 3,1985$, p. 76. Preuve addıtıonnelle de leur implicatıon importante dans la danse-theâtre, les Cabiers de theatre Jeu lui confient un dossier sur *la nouvelle danse" au Québec en 1984 ( $\left.\mathrm{n}^{\circ} 32\right)$

24. Voir entre autres les nos $73-74$ de la revue Alternatnves théatrales (juiller 2002), entièrement consacrés à la mise en scène des Aveugles; Hélène JACQUES, "Projections de la mort. Sur deux mises en scène de Denis Marleau», L'Annuaire thếtral, n $n^{\circ}$ 37, prınremps 2005, p. 113-127; Hélène JACQUES, «Un theâtre d'acteurs vidéographiques. Les Aveugles de Denis Marleau *, Intermédralutés, n6, pruntemps 2006, p. 79-94; Josette FÉRAL, « Denis Marleau. Une approche ludique et poétique ", Mise en scène et jeu de l'acteur, tome II, Montrél/Carnıères, Cahiers de théâtre Jeu/Lansman Éditeur, 1998, p. 185. 
nous invite à interroger le rôle du corps de l'acteur dans quelques-unes des pièces récentes de Marleau. Puisque son esthétique repose avant tout sur le dépouillement de la scène - et cela jusqu'à l'éviction complète des acteurs présents et vivant sur scène pendant la représentation - et sur une approche rigoureusement textocentriste, on oublie souvent le travail corporel de l'acteur marlusien au profit du travail vocal, celui-ci enregistré au préalable. En proposant une comparaison entre deux expériences théâtrales imaginées par Marleau à partir de l'œuvre de Maeterlinck - Les aveugles en 2002 et un spectacle présenté en Italie en 2004 à partir d'extraits de Pélléas et Mélisandre, de La mort de Tintagiles et des Aveugles -, Therrien prend le pouls du rôle de l'acteur dans la pratique de Marleau et y trouve un corps qui va de la nonaction à la disparition totale.

Tout comme Carbone 14, Robert Lepage (1957-...) s'est également donné son propre lieu de création - La Caserne à Québec, inaugurée en 1997 -, et c'est de là qu'opère cet homme de théâtre polyvalent et mondialement reconnu. Renommé pour ses spectacles ambitieux, épiques, collectifs et multidisciplinaires - on se rappellera entre autres La trilogie des dragons (1985), Les plaques tectoniques (1988), Les sept branches de la rivière Ota (1994) et La géométrie des miracles (1998) -, Lepage crée aussi des spectacles solo plus intimes, dont La face cachée de la lune (2000) et Le projet Andersen (2005). Il monte aussi des pièces de répertoire, de Shakespeare (notamment au Royal National Theatre de Londres et au Tokyo Globe Theatre), de Strindberg (au Royal Dramatick Theatre de Stockholm), ainsi que quelques opéras. Enfant chéri de la critique, Lepage se fait pourtant critiquer, selon notre collaboratrice Karen Fricker, pour la manière dont il met en scène la différence culturelle. Après un historique des représentations de cette différence culturelle dans l'œuvre de Lepage, Fricker revient soigneusement sur la place qu'elle a occupée dans la réception critique du spectacle-cabaret $Z u l u$ Time (2002), en examinant les lectures parfois divergentes de la critique francophone et anglophone. Fermement ancrée dans une perspective postcoloniale, l'analyse de Fricker propose un nouveau regard sur l'œuvre de Lepage et se joint au débat qui a fait couler beaucoup d'encre au Québec depuis peu, à la suite de la Commission Bouchard-Taylor sur les accommodements raisonnables.

\section{LES ARTS DE LA PISTE}

Enfin, le dossier se clôt sur un article qui porte sur un autre emblème québécois des arts de la scène, le Cirque du Soleil. Comme les metteurs en scène contemporains, les cirques québécois jouissent eux aussi de frontières 
de plus en plus indistinctes entre les arts de la scène, et bénéficient d'un réseau international solide qui leur donne une importante visibilité à l'étranger. De jeunes cirques comme Les 7 doigts de la main et le Cirque Éloize poursuivent des tournées en Europe et aux États-Unis, refoulant les limites du cirque proprement dit en alliant théâtre acrobatique, arts circassiens, performance, musique et poésie. Si ces jeunes organisations repoussent les limites génériques du cirque, le Cirque du Soleil - le plus reconnu au monde - fonde quant à lui son esthétique sur des corps qui doivent surmonter les limites naturelles, physiques et psychiques chaque fois qu'ils entrent sur la piste. En écho à l'article d'Ève Irène Therrien sur le corps marlusien, ma propre contribution aborde les usages du corps au Cirque du Soleil - le corps personnage, le corps performant et le corps charnel -, qui permettent aussi de mieux comprendre la relation complexe et tendue que le nouveau cirque entretient avec le cirque traditionnel.

À la toute fin de ce dossier thématique, comme dans chaque numéro de Globe, le lecteur trouvera deux rubriques dont la visée est d'effectuer un retour critique sur des questions d'actualité liées au thème du numéro. Explorant les assises conceptuelles du travail d'interprétation des pratiques scéniques dites "interartistiques» (danse-théâtre, théâtre technologique, nouvelles formes du cirque, etc.), Marie-Christine Lesage, dans sa "Note critique", effectue d'abord une traversée des publications savantes susceptibles d'offrir des balises dans la conceptualisation théorique des productions hybrides contemporaines. En même temps qu'elle rend compte des recherches engagées dans la réflexion interdisciplinaire, cette recension croisée d'ouvrages récents et moins récents a le grand mérite de désigner aussi les silences et les manques qui caractérisent cette production théorique. C'est pour combler ces lacunes que Lesage initie aussi le lecteur à quelques ouvrages consacrés à la performance, qui abordent plus directement l'aspect théorique de l'interdisciplinarité dans la pratique contemporaine de l'histoire de l'art. Dans la chronique "Perspective", Sylvain Schryburt propose quant à lui une réflexion critique sur l'état actuel de l'institution théâtrale québécoise, réflexion qui s'inscrit dans la foulée des deuxièmes États généraux du théâtre québécois, qui ont eu lieu à l'automne 2007. Volontiers polémique, il interroge les conséquences négatives d'une longue chaîne d'institutions qui, depuis les écoles de formation jusqu'aux organismes de financement, en passant par les réseaux de diffusion du théâtre, favorise le statu quo du milieu professionnel et freine l'essor de la relève thêâtrale, avec ce que cela implique sur le plan esthétique.

À tous, bonne lecture! 